\title{
Evaluación de riesgos psicosociales en el profesorado de la Universidad de Granada: Estudio de caso único
}

\author{
María Caballero Martínez y Lucía Fernández Madero. Universidad de Granada
}

\author{
Recepción: 12 de mayo de 2015 | Revisión: 17 de junio de 2015 | Aceptación/Publicación: 17 de julio de 2015 \\ Correspondencia: mariacaballero26@correo.ugr.es | luciafmadero@correo.ugr.es \\ Citar: Caballero, M. y Fernandez, L. (2015). Evaluación de riesgos psicosociales en el profesorado de la Universidad de Granada: \\ Estudio de caso único. ReiDoCrea, 4, 167-171.
}

Resumen: El estrés es considerado como uno de los riesgos psicosocial que mayor impacto tiene sobre la vida de las personas. Dado que en ocasiones el puesto de trabajo puede suponer una fuente de estrés y otros riesgos psicosociales, resulta de especial relevancia incidir sobre la necesidad de llevar a cabo una adecuada prevención de riesgos laborales, a fin de maximizar la calidad de vida de los trabajadores. De este modo, el presente informe evalúa los riesgos psicosociales en una profesora de la Universidad de Granada, utilizando para ello el ISTAS 21. Los resultados reflejan que las puntuaciones del sujeto en tres de las dimensiones evaluadas, Exigencias psicológicas, Inseguridad y Doble presencia, suponen un potencial riesgo para su salud. Finalmente se analizan las causas de dichas puntuaciones y se proponen diferentes estrategias de intervención.

Palabras clave: Riesgos laborales, Riesgos psicosociales.

PSYCHOSOCIAL RISKS EVALUATION AMONG THE PROFESSORATE OF THE UNIVERSITY OF GRANADA: A SINGLE-CASE STUDY

ABSTRACT: Thus, this report evaluates the psycho-social risks in a University of Granada Professor using the methodology ISTAS 21. The results show that the points that the subject scored in three of the evaluated dimensions (Psychological Demands, Insecurity and Dual Presence) are a potential risk for their health. Finally, the causes of said points are analyzed and different strategies of intervention are suggested.

Keywords: Occupational hazards, Psychosocial risks.

\section{Introducción}

Los riesgos laborales son los elementos en el puesto de trabajo generadores de estrés que impactan en la calidad de vida laboral y en el desempeño profesional (TeránRosero y Botero-Álvarez, 2012). Tradicionalmente, los riesgos laborales se han asociado a amenazas físicas, químicas y ambientales, pero actualmente cobran gran importancia los riesgos psicosociales, aquellos factores de estrés que pueden alterar y desequilibrar los recursos y las capacidades de la persona para manejar y responder al flujo de la actividad derivada del trabajo, dañando no sólo su salud física, sino también la psicológica. (Moreno-Jiménez, 2011).

Los estresores laborales se clasifican según si son relativos al ambiente físico, al contenido de la tarea o a la organización, de manera que, según las diferencias individuales, dañarán más o menos al trabajador. Aparte de las consecuencias físicas del estrés laboral, los daños en salud mental son muy relevantes, ya que suelen aparecer problemas de concentración, de atención, bloqueos, hipersensibilidad, incapacidad en la toma de decisiones, dependencia de fármacos, trastornos del sueño, depresión y ansiedad, de manera que disminuye la eficiencia del trabajador, así como su calidad de vida, y las repercusiones alcanzan también a la propia organización (Del Hoyo-Delgado, 2014).

La prevención de riesgos laborales, por tanto, tiene como objetivo la promoción de la mejora de las condiciones de trabajo para elevar el nivel de protección de la salud y la seguridad de los trabajadores y evitar que haya accidentes o daños para la salud de las personas (Junta de Andalucía, 2015). 
Entre los métodos de evaluación de los riesgos psicosociales, destaca el CoPsoQ (la versión española es ISTAS 21), un cuestionario que pretende la investigación, evaluación y prevención de los riesgos psicosociales.

El cuestionario ISTAS 21 ofrece una puntuación numérica que refleja la situación de exposición (verde, amarillo o rojo) en la que se encuentra el sujeto a cada una de las seis dimensiones psicosociales que evalúa: exigencias psicológicas, trabajo activo y posibilidades de desarrollo, apoyo social y calidad de liderazgo, inseguridad, doble presencia y estima.

En concreto, las Exigencias psicológicas evalúan tanto las exigencias cuantitativas como las cognitivas y emocionales referidas a las demandas del puesto de trabajo; la dimensión de Trabajo activo y posibilidades de desarrollo evalúa las perspectivas de crecimiento personal en el ámbito laboral, así como la percepción de influencia del propio sujeto en su trabajo mediante, por ejemplo, el grado de control en el tiempo de trabajo; por su parte, la dimensión de Inseguridad se incluye junto con la Estima en la dimensión Falta de Compensaciones, en la cual se hace referencia a los posibles cambios en el futuro a nivel laboral; en cuanto al Apoyo social y calidad de liderazgo, cabe destacar que evalúa la identificación con el rol desempeñado en el puesto de trabajo, la satisfacción con el clima laboral y la gestión de liderazgo; por último, la dimensión de Doble presencia refleja el estrés que conlleva el hecho de no poder dedicar el tiempo deseado a la familia y el hogar (Moncada, Llorens, Navarro y Kristensen, 2005).

Cada uno de los tres intervalos anteriormente mencionados clasifica a la población ocupada de referencia en tres grupos exactamente iguales: el intervalo verde incluye a la población de referencia con un nivel de exposición psicosocial más favorable para la salud; el intervalo amarillo refleja un nivel de exposición psicosocial intermedio y el intervalo rojo incluye a la población de referencia con un nivel de exposición psicosocial más desfavorable para la salud (Lluís, Serrano, Kristensen y Martínez, 2005).

El objetivo principal de la investigación fue medir en el sujeto los factores de riesgo psicosociales asociados al estrés laboral en su puesto de trabajo, en este caso, docente en la Universidad de Granada, así como proporcionar una propuesta de intervención acorde a la casuística personal para hacer frente a los posibles factores de riesgo.

\section{Método}

El cuestionario ISTAS 21 ha sido aplicado a una profesora perteneciente al departamento de Psicología Social de la facultad de Psicología de Granada; no obstante, también es docente en la facultad de Ciencias de la Educación, concretamente en la titulación de Educación Social. Además, dirige los Trabajos Fin de Grado de cuatro estudiantes, así como una Tesis Doctoral.

De acuerdo con ello, cabe destacar que su horario laboral presencial incluye además de los horarios de clase de estas asignaturas, las horas de tutorías correspondientes a cada una de ellas. Por consiguiente, su jornada laboral se reparte tanto en horarios de mañana como de tarde.

En la actualidad, está casada y tiene dos hijos, de seis años y veintidós meses respectivamente. Aunque ella misma se encarga de realizar algunas tareas del hogar, es importante señalar que cuenta con servicio doméstico varios días a la semana. 
En el mes de marzo de 2015 se le solicitó su colaboración para completar el cuestionario ISTAS 21. Dado su consentimiento, comenzó a contestarlo en su despacho de la facultad de Psicología, únicamente en presencia de los investigadores. Después de cumplimentarlo, respondió algunas preguntas con respecto a las dimensiones más destacadas del cuestionario. De esta forma, la duración total de la aplicación del ISTAS 21 fue de 15 minutos. Por último, se le agradeció su participación.

\section{Resultados}

Una vez corregido el cuestionario, se obtuvieron las puntuaciones del sujeto en cada una de las dimensiones anteriormente descritas. Dichos resultados se pueden consultar en la TABLA 1.

\begin{tabular}{|c|c|c|c|c|}
\hline \multicolumn{5}{|c|}{ Tabla 1 } \\
\hline Resultados obtenidos en la aplicación del Istas 21 \\
\hline APARTADO & DIMENSIÓN PSICOSOCIAL & VERDE & AMARILLO & ROJO \\
\hline $\mathbf{1}$ & Exigencias psicológicas & & & 18 \\
\hline $\mathbf{2}$ & Trabajo activo y posibilidades de desarrollo & 36 & & \\
\hline $\mathbf{3}$ & Inseguridad & & & 7 \\
\hline $\mathbf{4}$ & Apoyo social y calidad de liderazgo & 32 & & \\
\hline $\mathbf{5}$ & Doble presencia & & & 10 \\
\hline $\mathbf{6}$ & Estima & & 12 & \\
\hline
\end{tabular}

En la tabla se puede observar que el sujeto ha obtenido una puntuación de 18 en la dimensión de Exigencias Psicológicas; por su parte, puntúa 36 en la dimensión de Trabajo activo y posibilidades de desarrollo; del mismo modo, en la dimensión de Inseguridad tiene una puntuación de 7; en cuanto a la dimensión de Apoyo social y calidad de liderazgo, la puntuación obtenida es de 32; por otro lado, puntúa 10 en la dimensión de Doble presencia; y finalmente, el sujeto ha puntuado 12 en la dimensión de Estima.

En consecuencia, las dimensiones en las que el sujeto ha obtenido puntuaciones que no se consideran de riesgo para su salud son: Trabajo activo y posibilidades de desarrollo, Apoyo social y calidad del liderazgo (ambas situadas en el intervalo verde) y Estima (cuya puntuación se encuentra en el intervalo amarillo); mientras que las dimensiones de Exigencias psicológicas, Inseguridad y Doble presencia (situadas en el intervalo rojo) sí son consideradas como posibles factores de riesgo para su salud.

\section{Discusión}

En base a los resultados obtenidos en el presente estudio, cabe destacar que, en primer lugar, se han pretendido analizar las puntuaciones de las distintas dimensiones estudiadas que suponen un potencial riesgo para la salud del sujeto, esto es, aquellas que se corresponden con el intervalo rojo: Exigencias psicológicas, Inseguridad y Doble presencia.

La primera dimensión llamativa es la referente a las Exigencias psicológicas. Es probable que esta puntuación haya sido alta debido a la elevada carga laboral que presenta el sujeto, tal y como ya se ha descrito con anterioridad. Además, cabe resaltar que la sobrecarga de trabajo puede estar, a su vez, influyendo negativamente en los recursos emocionales del sujeto.

Por su parte, en cuanto a la dimensión de Inseguridad cabe señalar que, entre los posibles cambios en el futuro a nivel laboral que el sujeto considera, destaca una 
especial preocupación por el cambio de horario, ya que esto repercutiría notablemente en la organización de su vida familiar.

Finalmente, es importante mencionar que el sujeto puntúa alto en la dimensión de Doble presencia debido al hecho de no poder dedicar el tiempo que a ella le gustaría a su familia, especialmente a sus dos hijos, que aún son muy pequeños. Concretamente, las tareas del hogar no son su preocupación principal porque, como ya se mencionó anteriormente, cuenta con ayuda doméstica. No obstante, afirma que muchas veces necesitaría estar más tiempo en casa para dedicarse plenamente a su familia, por ejemplo, ayudando a sus hijos con los deberes o acompañándoles a sus actividades extraescolares, entre otros.

Por tanto, en base a los resultados obtenidos, se proponen las siguientes estrategias de intervención específicas:

Planificación y organización del tiempo: Es importante que el sujeto haga planificaciones semanales de su horario laboral, así como de las horas de tutoría que debe permanecer en el despacho y de los trabajos que tenga que corregir o supervisar, de manera que no se le acumulen las tareas laborales y pueda aprovechar su tiempo en familia, sin estas preocupaciones.

Reestructuración cognitiva: Ante la carga laboral que necesariamente implica su trabajo, resulta relevante hacer un especial énfasis en los pensamientos catastrofistas asociados, evitando las preocupaciones poco fundamentadas en la realidad que aumentan el estrés laboral. Para ello, es útil plantear preguntas de realidad y probabilidad de ocurrencia ante esas situaciones hipotéticas negativas que guíen al sujeto hacia argumentos más realistas y, en consecuencia, menos estresantes.

Relajación: Para complementar la eficacia de la reestructuración cognitiva, adquiere una gran importancia enseñar al sujeto diversas técnicas de relajación que pueda poner en práctica ante la sensación de agobio en el trabajo. Concretamente, en primer lugar se utilizarían técnicas basadas en la relajación guiada por imaginación para más tarde pasar a la Técnica de Relajación Muscular Progresiva de Jacobson (1929). Con esta técnica, en primer lugar el sujeto debe aprender a reconocer la tensión muscular, realizando para ello ejercicios de tensión-relajación con distintos niveles de intensidad. A continuación, la atención se dedica exclusivamente a relajar el músculo, dejando que lentamente desaparezca la tensión. Seguidamente, se da paso a la relajación diferencial, esto es, mantener relajados los músculos que no están implicados funcionalmente en las actividades diarias del sujeto (Vila y Fernández-Santaella, 2004). De esta forma, se llegaría a automatizar el aprendizaje para poder relajarse de forma eficaz y rápida cuando aparezcan pensamientos negativos.

\section{Referencias}

Del Hoyo-Delgado, M.A. (2014). Estrés laboral. Madrid: Instituto Nacional de Seguridad e Higiene en el Trabajo. Ministerio de Trabajo y Asuntos Sociales. Recuperado de: http://www.insht.es/InshtWeb/Contenidos/Documentacion/FICHAS\%20DE\%20PUBLICACIONES/FONDO\%2 OHISTORICO/DOCUMENTOS\%20DIVULGATIVOS/DocDivulgativos/Fichero\%20pdf/Estres\%20laboral.pdf

Junta de Andalucía. (2015). En qué consiste la prevención de riesgos laborales. Instituto Nacional de Seguridad e Higiene en el Trabajo. http://www.juntadeandalucia.es/organismos/economiainnovacioncienciayempleo/areas/seguridadsalud/riesgos-laborales.html

Lluís, S. M., Serrano, C. L., Kristensen, T. S., y Martínez, S. V. (2005). NTP 703: El método COPSOQ (ISTAS21, PSQCAT21) de evaluación de riesgos psicosociales. Notas Técnicas de Prevención. 
Moreno, B. (2011). Factores y riesgos laborales psicosociales: conceptualización, historia y cambios actuales. Medicina y Seguridad del Trabajo, 57, 4-19.

Terán, A. C. y Botero, C. C. (2012). Riesgos psicosociales intralaborales en docencia. Revista Iberoamericana de Psicología: Ciencia y Tecnología, 5(2), 95-106.

Vila, J. y Fernández-Santaella, M. C. (2004). Desactivación fisiológica. En Vila, J. y Fernández-Santaella, M. C. (Eds.), Tratamientos Psicológicos: La Perspectiva Experimental, (pp. 71-85). Madrid: Ediciones Pirámide. 